\title{
Extending the Nash Solution to Choice Problems with Reference Points
}

\author{
by \\ Peter Sudhölter \\ and \\ José M. Zarzuelo
}

Discussion Papers on Business and Economics

No. 13/2012

\author{
FURTHER INFORMATION \\ Department of Business and Economics \\ Faculty of Social Sciences \\ University of Southern Denmark \\ Campusvej 55 \\ DK-5230 Odense M \\ Denmark \\ Tel.: +456550 3271 \\ Fax: +4565503237 \\ E-mail: 1ho@sam.sdu.dk \\ http://www.sdu.dk/ivoe
}

ISBN 978-87-91657-66-5 


\title{
Extending the Nash Solution to Choice Problems with Reference Points*
}

\author{
Peter Sudhölter ${ }^{\dagger} \quad$ José M. Zarzuelo
}

\begin{abstract}
In 1985 Aumann axiomatized the Shapley NTU value by non-emptiness, efficiency, unanimity, scale covariance, conditional additivity, and independence of irrelevant alternatives. We show that, when replacing unanimity by "unanimity for the grand coalition" and translation covariance, these axioms characterize the Nash solution on the the class of $n$-person choice problems with reference points. A classical bargaining problem consists of a convex feasible set that contains the disagreement point here called reference point. The feasible set of a choice problem does not necessarily contain the reference point and may not be convex. However, we assume that it satisfies some standard properties. Our result is robust so that the characterization is still valid for many subclasses of choice problems, among those is the class of classical bargaining problems. Moreover, we show that each of the employed axioms - including independence of irrelevant alternatives - may be logically independent of the remaining axioms.
\end{abstract}

Keywords: Bargaining problem, Nash set, Shapley NTU value JEL codes: C71, C78

\section{Introduction}

A bargaining problem on a set $N$ of $n$ agents consists of a pair $(S, d)$ where $S \subseteq \mathbb{R}^{N}$, the feasible set, is a nonempty comprehensive set of utility profiles and $d \in S$ is the disagreement point. Nash (1950) characterized the Nash solution on the class of bargaining problems with convex feasible sets by Pareto efficiency (EFF), equal treatment of equals, scale covariance (SCOV), and independence of irrelevant alternatives (IIA). Bargaining problems may be regarded as particular cooperative non transferable utility (NTU) games, and, hence, the Shapley NTU value

\footnotetext{
*Financial support from the Spanish Ministerio de Ciencia e Innovación under project ECO2009-11213, cofunded by the ERDF, is acknowledged.

${ }^{\dagger}$ Department of Business and Economics and COHERE, University of Southern Denmark, Campusvej 55, 5230 Odense M, Denmark. e-mail: psu@sam.sdu.dk

${ }^{\ddagger}$ Faculty of Economics and Business Administration, Basque Country University, Lehendakari Aguirre 83, 48015 Bilbao, Spain. e-mail: josemanuel.zarzuelo@ehu.es
} 
(see Shapley (1969)) is a generalization of the Nash solution. Aumann (1985) characterized the Shapley NTU value (on the class of NTU games with closed, non-leveled, and smooth feasible sets) by non-emptiness (NE), EFF, conditional additivity (CADD), unanimity (UNA), SCOV, and $^{1}$ IIA. For $n=2$, an NTU game is either a bargaining problem or a rationing problem, i.e., a pair $(S, c)$ where $S$ is an aforementioned feasible set and $c \in \mathbb{R}^{N}$, the profile of reference utilities, does not belong to $S$. For an extensive study of rationing problems and their Nash rationing solutions see Mariotti and Villar (2005).

Many authors investigated generalizations of the Nash solution for bargaining problems with non-convex feasible sets (see, e.g., Herrero (1989), Conley and Wilkie (1996), Zhou (1996), Mariotti (1998), and Peters and Vermeulen (2010)). We consider both, bargaining problems and rationing problems, and do not assume in general that a feasible set is convex.

Rubinstein and Zhou (1999) introduce "choice problems with reference points", i.e., pairs $(P, r)$ where $P$ is a set of feasible alternatives in some set $X$ and $r$ is a "reference point" in $X$. They do not intend to, e.g., model conflicting interests in a bargaining problem, and a choice problem may be a single decision maker problem in which various criteria play the role of the involved bargainers. In contrast to these authors, we specify these conflicting interests explicitly by considering the elements of $P$ and $r$ itself as utility profiles. Despite of these differences in the interpretations, bargaining and rationing problems have the same structure: They consist of a feasible set (of utility profiles) and a reference utility profile. Thus, we keep the name and call a pair $(P, r)$ where $P \subseteq \mathbb{R}^{N}$ is a feasible set and $r \in \mathbb{R}^{N}$ a choice problem (on $N$ ) with reference point $(r)$ as well. The Nash solution of a choice problem with reference point $(P, r)$ is the Shapley NTU value of the associated NTU game if $P$ is non-leveled, smooth, and convex: A Pareto efficient element $x$ of $P$ is a Shapley NTU value if and only if, up to some natural scaling, it is the Shapley value of the transferable utility TU game that arises when replacing $P$ by the half-space determined by the supporting hyperplane through $x$. Hence, the concept of Nash solution may be generalized to a choice problem $(P, r)$ with a non-convex (but still non-leveled and smooth) feasible set by replacing "supporting hyperplane" with "tangent hyperplane" where necessary. We show that suitable versions of the axioms used by Aumann may be used to characterize the aforementioned generalization of the Nash solution to several classes of choice problems with reference points. The axiom UNA requires for NTU games that the solution equally divides the available one unit to the players of $S$ in the unanimity game of any subcoalition $S$ of $N$. Thus, the unique unanimity game that may be regarded as a choice problem with reference point (in fact a bargaining problem) is the unanimity game of the grand coalition $N$. Hence, UNA and SCOV on choice problems do not longer imply translation covariance (TCOV) as in the case of NTU games. Thus, in our results TCOV is additionally employed.

The paper is organized as follows. In Section 2 the necessary notation is provided, the definitions are presented, and straightforward properties of the Nash solution are deduced or recalled. It is also shown that the Nash solution of a uniformly p-smooth (in the sense of Maschler and Owen (1992)) choice problem with reference point always exists.

\footnotetext{
${ }^{1}$ His additional axiom of "Closure Invariance" is not relevant for NTU games with closed feasible sets.
} 
Section 3 is devoted to the axiomatization of the Nash solution on uniformly p-smooth choice problems with reference points. It is shown that Aumann's (1985) characterization of the NTU Shapley value may be resembled: The Nash solution is characterized by NE, EFF, CADD, UNA, SCOV, TCOV, and IIA (see Theorem 3.1). In Section 4 it is shown by means of examples that each of the employed axioms is logically independent of the remaining six axioms.

Section 5 investigates the "robustness" of Theorem 3.1. It turns out that the characterization still holds on many interesting classes of choice problems with reference points. Indeed the result holds for many classes of choice problems with convex feasible sets; e.g., for the class of classical bargaining problems with non-leveled smooth convex feasible sets, the Nash solution is characterized by the first six axioms, i.e., IIA is not needed. Also it turns out that, if IIA is slightly modified, the set of uniformly p-smooth choice problems may be replaced by the set of $p$-smooth choice problems with a nonempty Nash solution. Finally, this section contains some remarks about the logical independence of the axioms that are employed in the aforementioned modifications of Theorem 3.1.

The appendix (Section 6) is devoted to show some technical results that are used in the preceding sections. Namely, it is shown that for any point $x$ of its boundary, any (uniformly) p-smooth feasible set contains a convex (uniformly) p-smooth feasible set that contains $x$.

\section{Preliminaries}

For a finite set $N$ we denote by $\mathbb{R}^{N}$ the set of all real functions on $N$. So $\mathbb{R}^{N}$ is the $|N|$ dimensional Euclidean space. (Here and in the sequel, if $D$ is a finite set, then $|D|$ denotes the cardinality of $D$.) For $x, y \in \mathbb{R}^{N}$ let $x \cdot y$ denote the scalar product, and we write $x \geqslant y$ if $x_{i} \geqslant y_{i}$ for all $i \in N$. Moreover, we write $x>y$ if $x \geqslant y$ and $x \neq y$ and we write $x \gg y$ if $x_{i}>y_{i}$ for all $i \in N$. We denote $\mathbb{R}_{+}^{N}=\left\{x \in \mathbb{R}^{N} \mid x \geqslant 0\right\}$ and $\mathbb{R}_{++}^{N}=\left\{x \in \mathbb{R}^{N} \mid x \gg 0\right\}$. For every $S \subseteq N$, the indicator function on $S$ is denoted by $\chi^{S} \in \mathbb{R}^{N}$, i.e.,

$$
\chi_{j}^{S}=\left\{\begin{array}{l}
1, \text { if } j \in S, \\
0, \text { if } j \in N \backslash S,
\end{array}\right.
$$

and $x(S)=x \cdot \chi^{S}=\sum_{i \in S} x_{i}$ for every $x \in \mathbb{R}^{N}$.

Let $N$ be a finite nonempty set. A choice problem of $N$ is a pair $(P, r)$ such that $r \in \mathbb{R}^{N}$ and $P \subseteq \mathbb{R}^{N}$ is feasible (for $N$ ), i.e.,

$$
\begin{gathered}
P \neq \emptyset, \mathbb{R}^{N} ; \\
P \text { is closed; } \\
P \text { is comprehensive: } \quad x \in P, y \in \mathbb{R}^{N}, y \leqslant x \Rightarrow y \in P .
\end{gathered}
$$

We recall the definition of "uniform positive smoothness" (see, e.g., Maschler and Owen (1989)).

Definition 2.1 Let $P \subseteq \mathbb{R}^{N}$ be feasible. Denote by $\partial P$ the boundary of $P$. The set $P$ is $\mathbf{\text { smooth }}$ if 
(1) at every $x \in \partial P$ there exists a unique tangent hyperplane $H^{x}=H^{x, P}$ to $P$;

(2) the mapping $\partial P \rightarrow \mathbb{R}_{+}^{N}, x \mapsto \lambda^{x}=\lambda^{x, P}$, is continuous, where $\lambda^{x} \in \mathbb{R}^{N}$ is the unique normal vector determined by the requirements $\lambda^{x}(N)=1$ and $H^{x}=\left\{y \in \mathbb{R}^{N} \mid \lambda^{x} \cdot y=\lambda^{x} \cdot x\right\}$.

Moreover, a smooth set $P$ is positively smooth (p-smooth) if $\lambda^{x, P} \gg 0$ for all $x \in \partial P$, and it is uniformly $p$-smooth if there exists $\delta>0$ such that $\lambda^{x, P} \gg \delta \chi^{N}$ for all $x \in \partial P$.

Thus, a choice problem $(P, r)$ is (uniformly $p$-)smooth if $P$ is (uniformly p-)smooth. Note that p-smoothness of $P$ implies non-levelness of $P$, an assumption that is widely used in the corresponding literature.

Remark 2.2 Let $N \neq \emptyset$ be finite and $\lambda \in \mathbb{R}_{++}^{N}$.

(1) Let $P \subseteq \mathbb{R}^{N}$ be feasible. Then there is a unique function $g=g^{\lambda, P}: \mathbb{R}^{N} \rightarrow \mathbb{R}$ that satisfies $x-g(x) \lambda \in \partial P$ for all $x \in \mathbb{R}^{N}$. The mapping $g$ is continuous and satisfies

$$
\begin{aligned}
P & =\left\{x \in \mathbb{R}^{N} \mid g(x) \leqslant 0\right\} ; \\
g(x+t \lambda) & =g(x)+t \forall x \in \mathbb{R}^{N} \forall t \in \mathbb{R} ; \\
g & \text { is nondecreasing; } \\
P \text { is smooth } & \Rightarrow \nabla g(x)=\frac{\lambda^{x-g(x) \lambda, P}}{\lambda \cdot \lambda^{x-g(x) \lambda, P}} \forall x \in \mathbb{R}^{N},
\end{aligned}
$$

where, for any differentiable real function $f: \mathbb{R}^{N} \rightarrow \mathbb{R}, \nabla f=\left(\frac{\partial f}{\partial x_{i}}\right)_{i \in N}$ denotes the gradient $^{2}$ of $f$.

(2) Let $g: \mathbb{R}^{N} \rightarrow \mathbb{R}$ be a continuous mapping that satisfies (2.5) and (2.6). Then the set $P^{g}=P$ defined by (2.4) is feasible and $g^{\lambda, P}=g$. Moreover, if $\nabla g$ is continuous, then $P \mathrm{~s}$ smooth, and $g$ is convex if and only if $P$ is convex.

Let $N$ be a finite nonempty set. We denote by $\Gamma_{N}$ the set of all p-smooth choice problems $(P, r)$ such that $P \subseteq \mathbb{R}^{N}$. A solution on $\Gamma \subseteq \Gamma_{N}$ is a mapping $\sigma$ that assigns to each $(P, r) \in \Gamma$ a set $\sigma(P, r) \subseteq P$. Some well-known properties of a solution $\sigma$ on $\Gamma$ are as follows. The solution $\sigma$ satisfies

(1) non-emptiness (NE) if $\sigma(P, r) \neq \emptyset \forall(P, r) \in \Gamma$;

(2) efficiency (EFF) if $\sigma(P, r) \subseteq \partial P \forall(P, r) \in \Gamma$;

(3) conditional additivity $(\mathrm{CADD})$ if $\left(P^{i}, r^{i}\right) \in \Gamma, i \in\{1,2,3\}, P^{3}=P^{1}+P^{2}$, and ${ }^{3} r^{3}=r^{1}+r^{2}$ imply that

$$
\sigma\left(P^{3}, r^{3}\right) \supseteq\left(\sigma\left(P^{1}, r^{1}\right)+\sigma\left(P^{2}, r^{2}\right)\right) \cap \partial P^{3} ;
$$

\footnotetext{
${ }^{2}$ By (2) of Definition 2.1, the function $g^{\lambda, P}$ is $\mathrm{C}^{1}$ provided that $P$ is smooth.

${ }^{3}$ Whenever applied to sets, the "+" denotes the "Minkowski sum".
} 
(4) unanimity (UNA) if $\left(U^{N}, 0\right) \in \Gamma$ implies $\sigma\left(U^{N}, 0\right)=\left\{\frac{1}{|N|} \chi^{N}\right\}$, where

$$
U^{N}=\left\{x \in \mathbb{R}^{N} \mid x(N) \leqslant 1\right\}
$$

(5) scale covariance (SCOV) if for all $(P, r) \in \Gamma$ and for all $\lambda \in \mathbb{R}_{++}^{N}$ such that $^{4}(\lambda * P, \lambda * r) \in \Gamma$, $\sigma(\lambda * P, \lambda * r)=\lambda * \sigma(P, r)$

(6) translation covariance (TCOV) if for all $(P, r) \in \Gamma$ and $y \in \mathbb{R}^{N}$ such that $(P+\{y\}, r+y) \in$ $\Gamma, \sigma(P+\{y\}, r+y)=\sigma(P, r)+\{y\}$;

(7) independence of irrelevant alternatives (IIA) if $(P, r),\left(P^{\prime}, r\right) \in \Gamma, P \subseteq P^{\prime}$ imply that $\sigma(P, r) \supseteq \sigma\left(P^{\prime}, r\right) \cap P$.

(8) independence of irrelevant expansions (IIE) if $(P, r),\left(P^{\prime}, r\right) \in \Gamma, P \subseteq P^{\prime}$ imply that $\sigma\left(P^{\prime}, r\right) \supseteq \sigma(P, r) \cap \partial P^{\prime}$.

Definition 2.3 Let $(P, r) \in \Gamma_{N}$. The (generalized) Nash solution of $(P, r)$, denoted by $\phi(P, r)$, is defined by

$$
\phi(P, r)=\left\{x \in \partial P \mid\left(x_{i}-r_{i}\right) \lambda_{i}^{x, P}=\left(x_{j}-r_{j}\right) \lambda_{j}^{x, P} \forall i, j \in N\right\} .
$$

Remark 2.4 Let $(P, r) \in \Gamma_{N}$. If $r \notin P$, then

$$
\phi(P, r)=\left\{x \in \partial P \mid x \leqslant r, \prod_{i \in N}\left(r_{i}-x_{i}\right) \geqslant \prod_{i \in N}\left(r_{i}-y_{i}\right) \forall y \in H^{x, P} \cap\left\{z \in \mathbb{R}^{N} \mid z \leqslant r\right\}\right\} .
$$

If $r \in P$, then

$$
\phi(P, r)=\left\{x \in \partial P \mid x \geqslant r, \prod_{i \in N}\left(x_{i}-r_{i}\right) \geqslant \prod_{i \in N}\left(y_{i}-r_{i}\right) \forall y \in H^{x, P} \cap\left\{z \in \mathbb{R}^{N} \mid z \geqslant r\right\}\right\} .
$$

Remark 2.5 Let $(P, r) \in \Gamma_{N}$. For $x \in \partial P$ define the TU game $(N, v)=\left(N, v_{r}^{x, P}\right)$ by $v(N)=$ $\lambda^{x, P} \cdot x$ and $v(S)=\lambda_{S}^{x, P} \cdot r_{S}$ for all $S \varsubsetneqq N$. Note that $x \in \phi(P, r)$ if and only if $\lambda^{x, P} * x$ is the Shapley value of $(N, v)$ (see Shapley (1969)).

In general, it is well-known that a generalized Nash solution may not exist even in the case that $N=\{1,2\}$. Indeed, if $(P, r)$ is defined by $r=0$ and $P=\left\{x \in \mathbb{R}^{N} \mid x_{1}<0\right.$ and $\left.x_{2} \leqslant-\frac{1}{x_{1}^{2}}\right\}$, then $\phi(P, r)=0$. Thus, we denote

$$
\begin{aligned}
\Gamma_{N}^{\phi} & =\left\{(P, r) \in \Gamma_{N} \mid \phi(P, r) \neq \emptyset\right\} \text { and } \\
\Gamma_{N}^{\text {uni }} & =\left\{(P, r) \in \Gamma_{N} \mid P \text { is uniformly p-smooth }\right\} .
\end{aligned}
$$

In order to show that $\Gamma_{N}^{\text {uni }} \subseteq \Gamma_{N}^{\phi}$, the following lemma is helpful.

\footnotetext{
${ }^{4}$ We use the notation $\lambda * x=\left(\lambda_{i} x_{i}\right)_{i \in N}$ for all $\lambda, x \in \mathbb{R}^{N}$.
} 
Lemma 2.6 Let $(P, r) \in \Gamma_{N}$.

(1) If $r \in \partial P$, then $\phi(P, r)=\{r\}$.

(2) If $r \notin P$ and $\max _{x \in P, x \leqslant r} \prod_{i \in N}\left(r_{i}-x_{i}\right)$ exists, then

$$
\emptyset \neq \arg \max \left\{\prod_{i \in N}\left(r_{i}-x_{i}\right) \mid x \in \partial P, x \leqslant r\right\} \subseteq \phi(P, r) \subseteq\{x \in \partial P \mid x \ll r\} .
$$

(3) If $r$ is in the interior of $P$ and $\max _{x \in P, x \geqslant r} \prod_{i \in N}\left(x_{i}-r_{i}\right)$ exists, then

$$
\emptyset \neq \arg \max \left\{\prod_{i \in N}\left(x_{i}-r_{i}\right) \mid x \in P, x \geqslant r\right\} \subseteq \phi(P, r) \subseteq\{x \in \partial P \mid x \gg r\} .
$$

Proof: The first statement is a straightforward consequence of the definition. The second inclusion in both of the remaining statements follows immediately from the definition of $\phi$. The first inclusions may be shown as follows. If $r \notin P$, then let $y \in \arg \max _{x \in P, x \leqslant r} \prod_{i \in N}\left(r_{i}-x_{i}\right)$. The hyperplane $\left\{z \in \mathbb{R}^{M} \mid \lambda \cdot z=\lambda \cdot y\right\}$ is a tangent to the hyperbola $\left\{z \in \mathbb{R}^{N} \mid r \gg\right.$ $\left.z, \prod_{i \in N}\left(r_{i}-z_{i}\right)=t\right\}$ so that $y \in \phi(P, r)$ by Remark 2.5. If $r$ is in the interior of $P$, then the proof is similar.

q.e.d.

Corollary 2.7 The generalized Nash solution of a uniformly p-smooth choice problem exists.

Proof: Let $(P, r) \in \Gamma_{N}^{\text {uni }}$. By (1) of Lemma 2.6 we may assume that $r \notin \partial P$. If $r \notin P$, then $\{x \in$ $\partial P \mid x \leqslant r\}$ is nonempty and compact by uniform p-smoothness so that $\max _{x \in P, x \leqslant r} \prod_{i \in N}\left(r_{i}-x_{i}\right)$ exists and (2) of Lemma 2.6 finishes the proof. In the remaining case we may employ (3) of Lemma 2.6.

q.e.d.

Remark 2.8 Without smoothness the Nash solution violates CADD (see Aumann (1985, Section 9, on particular Figure 1)). If $P$ and $Q$ are smooth feasible sets, $x \in P, y \in Q$, and $x+y \in \partial(P+Q)$, then $x \in \partial P, y \in \partial Q$, and the tangent hyperplanes at $x$ to $P$ and at $y$ to $Q$ are parallel, i.e., $\lambda^{x, P}=\lambda^{y, Q}$.

Corollary 2.9 The Nash solution on any $\Gamma \subseteq \Gamma_{N}^{\phi}$ satisfies NE, EFF, CADD, UNA, SCOV, TCOV, IIA, and IIE.

Remark 2.10 Let $(P, r) \in \Gamma_{N}$.

(1) Classically (see Nash (1950)) it was assumed that $r \in P$ and that $P$ is a convex set. In this case, $\phi(P, r)$ is a singleton, namely the vector $y$ that maximizes the Nash product, i.e., $\prod_{i \in N}\left(x_{i}-r_{i}\right)$, subject to $x \in P$ that satisfy $x \geqslant r$.

(2) Similarly, if $\mathbb{R}^{N} \backslash P$ is convex and $r \notin P$, then $\phi(P, x)$ consists of the unique vector that maximizes $\prod_{i \in N}\left(r_{i}-x_{i}\right)$ subject to $x \leqslant r$ and $x \in \partial P$ (see (2.10) of Remark 2.4). 


\section{Axiomatization on uniformly p-smooth choice problems}

The main result of this section is the following theorem.

Theorem 3.1 The Nash solution on $\Gamma_{N}^{u n i}$ is the unique solution that satisfies NE, EFF, CADD, UNA, SCOV, TCOV, and IIA.

Proof: By Corollary 2.9 we only have to show the uniqueness part. Let $\sigma$ be a solution on $\Gamma_{N}^{\text {uni }}$ that satisfies the desired axioms. Let $(P, r) \in \Gamma_{N}^{\text {uni }}$. It remains to show that $\sigma(P, r)=\phi(P, r)$.

We consider first the following special cases.

(1) $P=\left\{x \in \mathbb{R}^{N} \mid x(N) \leqslant 0\right\}, r=0$ : Let $x \in \sigma(P, r)$. By EFF, $x(N)=0$. By CADD, $x+\frac{1}{|N|} \chi^{N} \in \sigma\left(U^{N}, 0\right)$. By UNA, $x=0$. By NE, $\sigma(P, r)=\{0\}=\phi(P, r)$.

(2) There exists $\lambda \in \mathbb{R}_{++}^{N}$ such that $P=\left\{x \in \mathbb{R}^{N} \mid \lambda \cdot x \leqslant \lambda \cdot r\right\}$. In this case $\sigma(P, r)=$ $\phi(P, r)=\{r\}$ by Case 1 , SCOV, and TCOV.

(3) There exist $\lambda \in \mathbb{R}_{++}^{N}$ and $c>\lambda \cdot r$ such that $P=\left\{x \in \mathbb{R}^{N} \mid \lambda \cdot x \leqslant c\right\}$. Let

$$
P^{\prime}=\left\{x \in \mathbb{R}^{N} \mid \lambda \cdot x \leqslant c-\lambda \cdot r\right\} .
$$

By SCOV and UNA, $\sigma\left(P^{\prime}, 0\right)=\phi\left(P^{\prime}, 0\right)=\left\{\left(\frac{c-\lambda \cdot r}{|N| \lambda_{i}}\right)_{i \in N}\right\}$. By TCOV, $\sigma(P, r)=\sigma\left(P^{\prime}, 0\right)+$ $\{r\}=\phi(P, r)$.

(4) There exist $\lambda \in \mathbb{R}_{++}^{N}$ and $c<\lambda \cdot r$ such that $P=\left\{x \in \mathbb{R}^{N} \mid \lambda \cdot x \leqslant c\right\}$. Let

$$
P^{\prime}=\left\{x \in \mathbb{R}^{N} \mid \lambda \cdot x \leqslant-c\right\} \text { and } P^{\prime \prime}=\left\{x \in \mathbb{R}^{N} \mid \lambda \cdot x \leqslant 0\right\} .
$$

By EFF and CAD, $\sigma\left(P^{\prime},-r\right)+\sigma(P, r) \subseteq \sigma\left(P^{\prime \prime}, 0\right)$. By Case 1 and SCOV, $\sigma\left(P^{\prime \prime}, 0\right)=$ $\{0\}=\phi\left(P^{\prime \prime}, 0\right)$. By Case 3 and NE, $\sigma(P, r)=-\sigma\left(P^{\prime},-r\right)=\phi(P, r)$.

(5) $P$ is convex. Let $y \in \sigma(P, r)$. By EFF, $y \in \partial P$. Let $\lambda=\lambda^{y, P}$. Then $\lambda \cdot y \geqslant \lambda \cdot x$ for all $x \in P$ by convexity. Let

$$
P^{\prime}=\left\{x \in \mathbb{R}^{N} \mid \lambda \cdot x \leqslant \lambda \cdot y\right\} \text { and } P^{\prime \prime}=\left\{x \in \mathbb{R}^{N} \mid \lambda \cdot x \leqslant 0\right\} .
$$

By Cases $(2)-(4), \sigma\left(P^{\prime \prime}, 0\right)=\{0\}=\phi\left(P^{\prime \prime}, 0\right)$ and $\sigma\left(P^{\prime}, r\right)=\phi\left(P^{\prime}, r\right)$ is a singleton. By CADD, $\{y\}+\sigma\left(P^{\prime \prime}, 0\right) \subseteq \sigma\left(P^{\prime}, r\right)$ so that $y \in \phi(P, r)$ by IIA of $\phi$. The opposite inclusion is shown similarly by interchanging the roles of $\sigma$ and $\phi$.

(6) $\mathbb{R}^{N} \backslash P$ is convex. Let $y \in \sigma(P, r)$. By EFF, $y \in \partial P$. Let $\lambda=\lambda^{y, P}$ and $P^{\prime}=\left\{x \in \mathbb{R}^{N} \mid\right.$ $\lambda \cdot x \leqslant \lambda \cdot y\}$. By IIA and Cases $(2)-(4), y \in \sigma\left(P^{\prime}, r\right)=\phi\left(P^{\prime}, r\right)$. By IIE of $\phi, y \in \phi(P, r)$. In order to show that $\phi(P, r) \subseteq \sigma(P, r)$, let $z \in \phi(P, r)$. If $r$ is not in the interior of $P$, then, by Lemma $2.6(1)$ or Remark $2.10(2),|\phi(P, r)|=1$ so $z \in \sigma(P, r)$ by NE. 
Hence, we may assume that $r$ is in the interior of $P$. It is sufficient to find $\left(P^{\prime}, r\right),\left(P^{\prime \prime}, 0\right) \in$ $\Gamma_{N}^{\text {uni }}$ such that $0 \in \partial P^{\prime \prime}, \mathbb{R}^{N} \backslash P^{\prime \prime}$ is convex, $z \in \partial P^{\prime}, P^{\prime}$ is convex, $P^{\prime} \subseteq P$, and $P=$ $P^{\prime}+P^{\prime \prime}$. Indeed, as 0 is not in the interior of $P^{\prime \prime},\{0\}=\phi\left(P^{\prime \prime}, 0\right)=\sigma\left(P^{\prime \prime}, 0\right)$. Moreover, $z \in \phi\left(P^{\prime}, r\right)=\sigma\left(P^{\prime}, r\right)$ by IIE of $\phi$ and Case (5) so that $y \in \sigma(P, r)$ by CADD. Now, we shall construct $P^{\prime}$ and $P^{\prime \prime}$. Let $G^{P^{\prime}}, G^{P^{\prime \prime}}: \mathbb{R}^{N} \rightarrow \mathbb{R}^{N}$ be defined by

$$
G^{P^{\prime}}(x)=2 y-x, G^{P^{\prime \prime}}(x)=2 x-2 y \forall x \in \mathbb{R}^{N}
$$

and define

$$
P^{\prime}=\left\{z \in \mathbb{R}^{N} \mid \exists x \in \partial P: z \leqslant G^{P^{\prime}}(x)\right\}, P^{\prime \prime}=\left\{z \in \mathbb{R}^{N} \mid \exists x \in \partial P: z \leqslant G^{P^{\prime \prime}}(x)\right\} .
$$

With $\lambda=\lambda^{y, P}$ and $g=g^{\lambda, P}$ it may easily be deduced that, for all $x \in \mathbb{R}^{N}$,

$$
g^{\lambda, P^{\prime}}(x)=-g(2 y-x) \text { and } g^{\lambda, P^{\prime \prime}}(x)=2 g\left(\frac{x}{2}+y\right)
$$

so that $\nabla g^{\lambda, P^{\prime}}(x)=\nabla g(2 y-x)$ and $\nabla g^{\lambda, P^{\prime \prime}}(x)=\nabla g\left(\frac{x}{2}+y\right)$. Hence, $P^{\prime}$ and $P^{\prime \prime}$ are uniformly p-smooth by Remark 2.2. It is straightforward to verify that $P^{\prime}$ and $P^{\prime \prime}$ satisfy the desired properties.

(7) Now we are able to consider the general case and prove that $\sigma(P, r)=\phi(P, r)$.

(a) Let $y \in \sigma(P, r)$. By EFF and Corollary 6.3 there exists $(\widetilde{P}, r) \in \Gamma_{N}^{\text {uni }}$ such that $\widetilde{P}$ is convex, $y \in \partial \widetilde{P}$, and $\widetilde{P} \subseteq P$. By IIA, $y \in \sigma(\widetilde{P}, r)$. By Case $(5), y \in \phi(\widetilde{P}, r)$. By IIE of $\phi, y \in \phi(P, r)$.

(b) In order to show the opposite inclusion, let $z \in \phi(P, r)$. By EFF, $z \in \partial P$. By Corollary 6.3 there exists $\left(P^{\prime},-r\right) \in \Gamma_{N}^{\text {uni }}$ such that $P^{\prime}$ is convex, $P^{\prime} \subseteq-\left(\mathbb{R}^{N} \backslash P\right)$, and $-z \in P^{\prime}$. Let $P^{\prime \prime}=-\left(\mathbb{R}^{N} \backslash P^{\prime}\right)$. By IIE of $\phi, z \in \phi\left(P^{\prime \prime}, r\right)$. By Case (6), $z \in \sigma\left(P^{\prime \prime}, r\right)$. By IIA, $z \in \sigma(P, r)$.

q.e.d.

\section{On the Logical Independence of the Axioms}

The following solution, denoted by $\widehat{\phi}$, will be useful. For $(P, r) \in \Gamma_{N}^{u n i}$ let

$$
\widehat{\phi}(P, r)= \begin{cases}\phi(P, r) & \text { if } r \in P, \\ \arg \max \left\{\prod_{i \in N}\left(r_{i}-x_{i}\right) \mid x \in \partial P, x \leqslant r\right\} & \text {,if } r \notin P .\end{cases}
$$

Then $\widehat{\phi}$ satisfies NE by Lemma 2.6. In view of and EFF and UNA, because $\widehat{\phi}$ is a nonempty subsolution of $\phi$. It is straightforward to verify that $\widehat{\phi}$ satisfies SCOV, TCOV, and IIE. The following example shows that $\widehat{\phi}$ does not satisfy IIA provided that $|N| \geqslant 2$.

Example 4.1 Let $|N| \geqslant 2$ and $X=\left\{x \in \mathbb{R}^{N} \mid x \leqslant 0, \prod_{i \in N}\left(-x_{i}\right) \geqslant 1\right\}$. Then $X$ is a p-smooth feasible set. If $Y=\left\{y \in \partial X \mid y_{i} \geqslant-2\right\}$, then $Y \neq \emptyset$ so that $P=\left\{z \in \mathbb{R}^{N} \mid \lambda^{y, X} \cdot z \leqslant\right.$ $\left.\lambda^{y, X} \cdot y \forall y \in Y\right\}$ is uniformly p-smooth. Let $r=0$. We may easily deduce that $\phi(P, r)=Y$. 
Let $P^{\prime}=P-\left\{\chi^{N}\right\}$. By symmetry of $P, \phi\left(P^{\prime}, r\right) \ni-2 \chi^{N}$. Let $i \in N$. Define $\widehat{x}$ by $\widehat{x}_{j}=-3$ and $\widehat{x}_{i}=-1-\frac{1}{2^{|N|-1}}$ and observe that $\widehat{x} \in \partial P^{\prime}$. However, $\prod_{i \in N}-\widehat{x}_{i}=3^{n-1}+\frac{3^{n-1}}{2^{n-1}}>2^{n}=\prod_{i \in N} 2$ so that $-2 \chi^{N} \notin \widehat{\phi}\left(P^{\prime}, r\right)$. For sketches of $P$ and $P^{\prime}$ in the case $|N|=2$ see Figure 4.1.

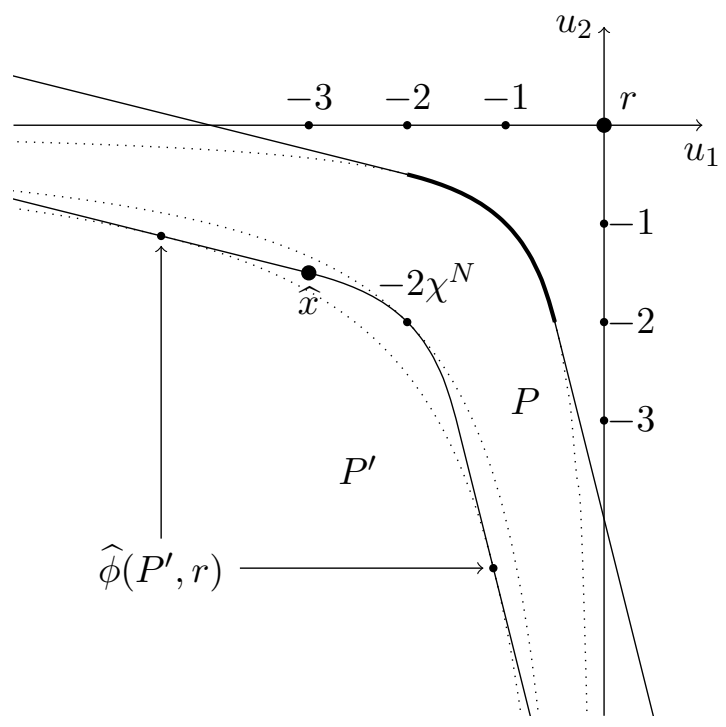

Figure 4.1: Sketch of the uniformly p-smooth feasible sets $P$ and $P^{\prime}$

We now show that $\widehat{\phi}$ satisfies CADD.

Lemma 4.2 The solution $\widehat{\phi}$ on $\Gamma_{N}^{u n i}$ satisfies $\mathrm{CADD}$.

Proof: For $i \in\{1,2\}$, let $\left(P^{i}, r^{i}\right) \in \Gamma_{N}^{\text {uni }}, x^{i} \in \widehat{\phi}\left(P^{i}, r^{i}\right)$ such that, with $P=P^{1}+P^{2}, r=r^{1}+r^{2}$, and $x=x^{1}+x^{2},(P, r) \in \Gamma_{N}^{\text {uni }}$ and $x \in \partial P$. By CADD of $\phi, x \in \phi(P, r)$. It remains to show that $x \in \widehat{\phi}(P, r)$. If $r \in P$, then the proof is finished.

Hence, we may assume that $r \notin P$. As $x \in \partial P, \lambda^{x^{1}, P^{1}}=\lambda^{x^{2}, P^{2}}=\lambda^{x, P}$. By Remark 2.5, there exists $c \in \mathbb{R}$ such that $\left(r^{2}-x^{2}\right)=c\left(r^{1}-x^{1}\right)$. By Lemma $2.6, x^{1} \ll r^{1}$ or $x^{2} \ll r^{2}$. Without loss of generality we may assume that $x^{1} \ll r^{1}$. By definition of $\widehat{\phi}$,

$$
P^{1} \supseteq\left\{z \in \mathbb{R}^{N} \mid z \ll r^{1}, \prod_{i \in N}\left(r_{i}^{1}-z_{i}\right) \geqslant \prod_{i \in N}\left(r_{i}^{1}-x_{i}^{1}\right)\right\}=: Z^{1}
$$

Let $Z=\left\{z \in \mathbb{R}^{N} \mid z \ll r, \prod_{i \in N}\left(r_{i}-z_{i}\right) \geqslant \prod_{i \in N}\left(r_{i}-x_{i}\right)\right\}$. Two cases may occur:

(1) $x^{2} \geqslant r^{2}$. By (4.1), $P \supseteq\left\{x^{2}\right\}+Z^{1}$. Let $z \in Z$ and define $z^{1}=z-x^{2}$. It suffices to show that $z^{1} \in Z^{1}$. Now, $z^{1} \ll r^{1}$, because $x^{2} \geqslant r^{2}$ and $z \ll r$. As $r \gg x,-1<c \leqslant 0$. With $a=r-z, b=r-x$, and $\alpha=-\frac{c}{1+c}$, we receive

$$
r^{1}-z^{1}=a+\alpha b, r^{1}-x^{1}=(1+\alpha) b, a, b>0, \text { and } \alpha \geqslant 0 .
$$


Hence, it suffices to show the following implication:

$$
a, b \in \mathbb{R}_{++}^{N}, \prod_{i \in N} a_{i} \geqslant \prod_{i \in N} b_{i}, \alpha \geqslant 0 \Longrightarrow \prod_{i \in N}\left(a_{i}+\alpha b_{i}\right) \geqslant \prod_{i \in N}\left((1+\alpha) b_{i}\right) .
$$

Indeed, (4.2) may be proved by induction on $|N|$. If $|N|=1$, then (4.2) is obviously valid. Assume (4.2) is valid for $|N| \leqslant r$ for some $r \in \mathbb{N}$. If $|N|=r+1$, then we may assume that $a_{k}<b_{k}$ for some $k \in N$ and, hence, $a_{\ell}>b_{\ell}$ for some $\ell \in N$. Define $\widetilde{a} \in \mathbb{R}^{N}$ by $\widetilde{a}_{i}=a_{i}$ for all $i \in N \backslash\{k, \ell\}, \widetilde{a}_{k} \widetilde{a}_{\ell}=a_{k} a_{\ell}$, and

$$
\tilde{a}_{k}=\left\{\begin{array}{cl}
b_{k} & , \text { if } a_{k} a_{\ell} \leqslant b_{k} b_{\ell} \\
\frac{a_{k} a_{\ell}}{b_{\ell}} & , \text { otherwise. }
\end{array}\right.
$$

Then

$$
\widetilde{a} \gg 0, \prod_{i \in N} \widetilde{a}_{i}=\prod_{i \in N} a_{i} \text {, and } \prod_{i \in N}\left(\widetilde{a}_{i}+\alpha b_{i}\right) \leqslant \prod_{i \in N}\left(a_{i}+\alpha b_{i}\right) .
$$

The proof is finished by applying the inductive hypothesis, either to $N \backslash\{k\}$, if $\widetilde{a}_{k}=b_{k}$, or to $N \backslash\{\ell\}$ in the other case, i.e., if $\widetilde{a}_{\ell}=b_{\ell}$.

(2) $x^{2} \ll r^{2}$. Let

$$
Z^{2}=\left\{z \in \mathbb{R}^{N} \mid z \ll r^{2}, \prod_{i \in N}\left(r_{i}^{2}-z_{i}\right) \geqslant \prod_{i \in N}\left(r_{i}^{2}-x_{i}^{2}\right)\right\}
$$

By definition of $\widehat{\phi}, P^{2} \supseteq Z^{2}$. As $Z^{1}+Z^{2} \supseteq Z$, the proof is finished.

q.e.d.

The solution $\sigma^{i}, i=1, \ldots, 6$, shows that the $i$-th axiom in Theorem 3.1 is logically independent of the remaining axioms provided that $|N| \geqslant 2$. If $(P, r) \in \Gamma_{N}^{\text {uni }}$, then

$$
\begin{aligned}
& \sigma^{1}(P, r)=\left\{\begin{array}{cl}
\phi(P, r) & , \text { if } r \in P \backslash \partial P \\
\emptyset & , \text { otherwise; }
\end{array}\right. \\
& \sigma^{2}(P, r)=\left\{\begin{array}{cl}
\phi(P, r) & , \text { if } r \in P \backslash \partial P, \\
\phi(P, r)-\mathbb{R}_{+}^{N} & , \text { otherwise; }
\end{array}\right. \\
& \sigma^{3}(P, r)=\left\{\begin{array}{cl}
\phi(P, r) & , \text { if } r \in P \backslash \partial P, \\
\partial P & , \text { otherwise; }
\end{array}\right. \\
& \sigma^{4}(P, r)=\partial P \\
& \sigma^{5}(P, r)=\left\{x \in \partial P \mid x=r+t \chi^{N} \text { for some } t \in \mathbb{R}\right\} ; \\
& \sigma^{6}(P, r)=\phi(P, 0) \text {. }
\end{aligned}
$$




\section{$5 \quad$ Bargaining problems and p-smooth choice problems}

This section is devoted to some modifications of Theorem 3.1 showing the robustness of this axiomatization.

We first treat p-smooth choice problems with convex feasible sets. Let $\Gamma_{N}^{\text {con }}=\left\{(P, r) \in \Gamma_{N} \mid\right.$ $P$ is convex $\}$. A set $\Gamma \subseteq \Gamma_{N}^{c o n}$ is a feasible domain of convex choice problems if

(1) $\Gamma \subseteq \Gamma_{N}^{\phi}$;

(2) $\left(\left\{x \in \mathbb{R}^{N} \mid x(N) \leqslant c\right\}, r\right) \in \Gamma$ for all $c \in \mathbb{R}$ and all $r \in \mathbb{R}^{N}$ such that $r(N) \leqslant c$;

(3) If $(P, r) \in \Gamma$ and $\lambda \in \mathbb{R}_{++}^{N}$, then $(\lambda * P, \lambda * r) \in \Gamma$;

(4) If $(P, r) \in \Gamma$ and $x \in \partial P$, then $\left(\left\{y \in \mathbb{R}^{N} \mid \lambda^{x, P} \cdot y \leqslant \lambda^{x, P} \cdot x\right\}\right) \in \Gamma$.

A careful inspection of the cases (1) through (5) in the proof of Theorem 3.1 shows that this result holds when replacing $\Gamma_{N}^{\text {uni }}$ by any feasible domain of convex choice problems, i.e., we have the following proposition.

Proposition 5.1 Let $\Gamma \subseteq \Gamma_{N}^{c o n}$ be a feasible domain. The Nash solution on $\Gamma$ is the unique solution that satisfies NE, EFF, CADD, UNA, SCOV, TCOV, and IIA.

A choice problem with reference point $(P, r)$ is a bargaining problem if $r \in P$. In this case, we may interpret $r$ as the disagreement point and use the letter $d$ instead of $r$. A bargaining problem $(P, d)$ is proper if $d$ is an element of the interior of $P$.

A careful inspection of the proof of Theorem 3.1 shows that this result still holds if $\Gamma_{N}^{\text {uni is }}$ replaced by the subset of uniformly p-smooth bargaining problems on $N$.

Moreover, it should be noted that the set of bargaining problems $(P, d) \subseteq \Gamma_{N}$ such that $P$ is convex is a feasible domain so that Proposition 5.1 applies also to this set. However, in this case, we don't need IIA.

Theorem 5.2 Let $\Gamma \subseteq \Gamma_{N}^{\text {con }}$ consist of bargaining problems only. Then $\Gamma \subseteq \Gamma_{N}^{\phi}$ and if $\Gamma$ is feasible, then the Nash solution on $\Gamma$ is the unique solution that satisfies NE, EFF, CADD, UNA, SCOV, and TCOV.

Proof: Let $(P, d) \in \Gamma$. By $(1)$ of Remark 2.10, $\phi(P, d) \neq \emptyset$. In order to show the second statement, we may follow the first part of the proof of Theorem 3.1. Indeed, we may first literally the proof until and including case (3) because IIA is not employed in this part. Note that cases 4,6 , and 7 cannot occur. The first inclusion $\sigma(P, d) \subseteq \phi(P, d)$ of case (5) may be literally copied-it just refers to IIA of $\phi$. The part that shows the other inclusion with the help of IIA may now be circumvented, because by (1) of Remark $2.10, \phi(P, d)$ is a singleton. q.e.d. 
We now check if our result may be applied to $\Gamma_{N}^{\phi}$. If we want to adjust the proof of Theorem 3.1 suitably, we first notice that we have to replace Corollary 6.3 by Lemma 6.2 wherever it occurs. Unfortunately, the general case (7) may not be adjusted in a straightforward manner. In fact, if $P$ is not uniformly p-smooth, then the convex p-smooth choice problem $(\widetilde{P}, r)$ whose existence is guaranteed by Lemma 6.2 may not be uniformly p-smooth so that $\phi(\widetilde{P}, r)=\emptyset$ might be possible.

In order to overcome this problem we employ a slightly stronger version of IIA. For the sake of completeness, we also modify IIE. Let $\Gamma \subseteq \Gamma_{N}$ and $\sigma$ be a solution on $\Gamma$. Then $\sigma$ satisfies

(6') IIA' if $(P, r) \in \Gamma_{N},\left(P^{\prime}, r\right) \in \Gamma, P \subseteq P^{\prime}$, and $\sigma\left(P^{\prime}, r\right) \cap P \neq \emptyset$ imply that $(P, r) \in \Gamma$ and $\sigma\left(P^{\prime}, r\right) \cap P \subseteq \sigma(P, r)$;

(7') IIE' if $\left(P^{\prime}, r\right) \in \Gamma_{N},(P, r) \in \Gamma, P \subseteq P^{\prime}$, and $\sigma(P, r) \cap \partial P^{\prime} \neq \emptyset$ imply that $\left(P^{\prime}, r\right) \in \Gamma$ and $\sigma(P, r) \cap \partial P^{\prime} \subseteq \sigma\left(P^{\prime}, r\right)$.

Note that $\phi$ satisfies IIA' and IIE' on $\Gamma_{N}^{\phi}$. Moreover, if we replace IIA and IIE by the stronger versions wherever they occur in the proof of Theorem 3.1, then we receive the following result.

Corollary 5.3 On the set $\Gamma_{N}^{\phi}$ the Nash solution is the unique solution that satisfies NE, EFF, CADD, UNA, SCOV, TCOV, and IIA'.

Remark 5.4 (1) Corollary 5.3 still holds when $\Gamma_{N}^{\phi}$ is replaced by the subset of all bargaining problems in $\Gamma_{N}^{\phi}$.

(2) The solutions $\sigma^{i}, i=1, \ldots 5$, defined in Section 4 still show that the $i$-th axiom is logically independent of the remaining axioms in all foregoing characterizations of the Nash solution provided $|N| \geqslant 2$. Moreover, $s^{6}$ still violates TCOV and satisfies EFF, CADD, UNA, SCOV, and IIA' on all mentioned classes of choice problems. However, $\sigma^{6}$ also violates NE if $\Gamma$ contains all bargaining problems in $\Gamma_{N}^{\phi}$ so that, e.g., we don't know it TCOV is really needed in Corollary 5.3.

(3) The logical independence of IIA' in Corollary 5.3 for is an open problem.

(4) Our solution $\widehat{\phi}$ defined in 4 still shows that IIA is needed in Proposition 5.1 if the feasible domain consists, e.g., of all convex uniformly p-smooth choice problems. However, in general it is an open problem if IIA is really needed. Only in the case $|N|=2$ it is known that IIA is redundant if $\Gamma=\Gamma_{N}^{\text {con }} \cap \Gamma_{N}^{\phi}$ (see Peleg, Sudhölter, and Zarzuelo (2012, Theorem $3.1)$ ).

\section{Appendix}

The following remark is useful. 
Remark 6.1 Let $P, Q \in \mathbb{R}^{N}$ be convex such that $P, Q$, and $P+Q$ are feasible.

(1) If $P$ is smooth, then $P+Q$ is also smooth.

(2) If $P$ is smooth and, for any $y \in \partial Q$ and any $\lambda \in \mathbb{R}^{N}$ such that $\lambda>0$ and such that $H=\left\{y^{\prime} \in \mathbb{R}^{N} \mid \lambda \cdot y^{\prime}=\lambda \cdot y\right\}$ is a supporting hyperplane at $y$ of $Q, \lambda \gg 0$, then $P+Q$ is p-smooth.

(3) If $P$ is smooth and there exists $\varepsilon>0$ such that, for any $y \in \partial Q$ and any $\lambda \in \mathbb{R}^{N}$ such that $\lambda>0$ and $\lambda(N)=1$ and such that $H=\left\{y^{\prime} \in \mathbb{R}^{N} \mid \lambda \cdot y^{\prime}=\lambda \cdot y\right\}$ is a supporting hyperplane at $y$ of $Q, \lambda \gg \varepsilon \chi^{N}$, then $P+Q$ is uniformly p-smooth.

Indeed, in order to verify Remark 6.1, note that any $z \in \partial(P+Q)$ is of the form $z=x+y$ for suitable $x \in \partial P$ and $y \in \partial Q$ and if $H=\left\{z^{\prime} \in \mathbb{R}^{N} \mid \lambda \cdot z^{\prime}=\lambda \cdot z\right\}$ for some $\lambda \geqslant 0, \lambda \neq 0$, is a supporting hyperplane at $z$ to $P+Q$, then $\left\{x^{\prime} \in \mathbb{R}^{N} \mid \lambda \cdot x^{\prime}=\lambda \cdot x\right\}$ is a supporting hyperplane of $P$ at $x$ so that $\lambda$ is proportional to $\lambda^{x, P}$ and $\left\{y^{\prime} \in \mathbb{R}^{N} \mid \lambda \cdot y^{\prime}=\lambda \cdot y\right\}$ is a supporting hyperplane at $y$ to $Q$. These observations immediately imply the desired statements.

Lemma 6.2 If $P \subseteq \mathbb{R}^{N}$ is a p-smooth feasible set and $y \in \partial P$, then there exists a p-smooth feasible set $\widetilde{P} \subseteq \mathbb{R}^{N}$ such that

$$
\begin{aligned}
\widetilde{P} & \text { is convex; } \\
y & \in \partial \widetilde{P} \\
\widetilde{P} & \subseteq P .
\end{aligned}
$$

Proof: For simplicity we may assume that $y=0$ (otherwise replace $P$ by $P-\{y\}$ and $y$ by 0 ). Moreover, we may assume that $\lambda^{y}=\lambda^{y, P}=\frac{1}{n} \chi^{N}$ (otherwise replace $P$ by $\lambda^{y} * P$ ). Let $g=g^{\lambda^{y}, P}$ and $X=H^{y, P}=\left\{x \in \mathbb{R}^{N} \mid \lambda^{y} \cdot x=0\right\}=\left\{x \in \mathbb{R}^{N} \mid x(N)=0\right\}$. Now we define $G: \mathbb{R}_{+} \rightarrow \mathbb{R}$ by the requirement that

$$
G(t)=\max \{\nabla g(r z) \cdot z \mid z \in X,\|z\|=1,0 \leqslant r \leqslant t\} \forall t \geqslant 0,
$$

and note that $G$ is well-defined by Remark 2.2. Moreover, $G(0)=0$ and $G$ is continuous. By the fundamental theorem of calculus there exists a function $\widehat{g}: \mathbb{R} \rightarrow \mathbb{R}$ such that $\widehat{g}^{\prime}=G$ and $\widehat{g}(0)=0$. As $G$ is monotonically increasing, $\widehat{g}$ is a convex function. For any $z \in X$ satisfying $\|z\|=1$, define $g_{z}: \mathbb{R} \rightarrow \mathbb{R}$ by $g_{z}(t)=g(t z)$ for all $t \in \mathbb{R}_{+}$. Then, for all $t \geqslant 0$,

$$
g_{z}^{\prime}(t)=\nabla g(t z) \cdot z \leqslant \widehat{g}^{\prime}(t) \text { and } g_{z}(0)=0 .
$$

Hence, we may conclude that

$$
g_{z}(t) \leqslant \widehat{g}(t) \forall t \in \mathbb{R}_{+} .
$$

Now we are ready to define $\bar{g}$ as follows: For $z \in \mathbb{R}^{N}$ let $x(z)=z-z(N) \lambda^{y}$. Then $x(z) \in X$ and we define $\bar{g}(z)=\widehat{g}(\|x(z)\|)+z(N)$ so that $\bar{g}\left(z+t \lambda^{y}\right)=\bar{g}(z)+t$ for $t \in \mathbb{R}$. By $(6.4), \bar{g}$ is symmetric in the sense that

$$
\bar{g}(z)=\bar{g}\left(z+\chi^{\{i\}}\left(z_{j}-z_{i}\right)+\chi^{\{j\}}\left(z_{i}-z_{j}\right)\right) \text { for all } z \in \mathbb{R}^{N} \text { and } i, j \in N .
$$


By (6.5) and (2.5),

$$
g(z) \leqslant \bar{g}(z) \forall z \in \mathbb{R}^{N}
$$

Define $\widehat{P}=\left\{z \in \mathbb{R}^{N} \mid \bar{g}(z) \leqslant 0\right\}-\mathbb{R}_{+}^{N}$. By (6.7) and comprehensiveness of $P, \widehat{P} \subseteq P$. Moreover, $y=0 \in \widehat{P}$. It is easy to check that, for all $z \in \mathbb{R}^{N}$ and all $i \in N$,

$$
\frac{\partial \bar{g}}{\partial x_{i}}(z)=\left\{\begin{array}{cl}
1 & , \text { if } x(z)=0 \\
\widehat{g}^{\prime}(\|x(z)\|) \frac{x_{i}(z)}{\|x(z)\|}+1 & , \text { if } x(z) \neq 0 .
\end{array}\right.
$$

As $\widehat{g}$ is a convex function, we conclude that $\widehat{P}$ is closed and convex. In order to show that $\widehat{P}$ is smooth, by convexity, it suffices to show that at each $z \in \partial \widehat{P}$ there exists a unique supporting hyperplane to $\widehat{P}$. Let $\lambda^{\prime} \in \mathbb{R}^{N}, \lambda^{\prime} \neq 0$, be some vector such that $\widehat{P} \subseteq\left\{z^{\prime} \in \mathbb{R}^{N} \mid \lambda^{\prime} \cdot z^{\prime} \leqslant \lambda^{\prime} \cdot z\right\}$. Then there exist $\widehat{x} \in \mathbb{R}^{N}$ with $\bar{g}(\widehat{x}) \leqslant 0$ and $x \in-\mathbb{R}_{+}^{N}$ such that $z=\widehat{x}+x$. Hence $\bar{g}(\widehat{x})=0$ and $x \in \partial\left(-\mathbb{R}_{+}^{N}\right)$. As $-\mathbb{R}_{+}^{N}$ is a cone, $\lambda^{\prime} \cdot x \leqslant 0$. As $\lambda^{\prime} \cdot \widehat{x}=\lambda^{\prime} \cdot(\widehat{x}+0 x) \leqslant \lambda^{\prime} \cdot z=\lambda^{\prime} \cdot \widehat{x}+\lambda^{\prime} \cdot x$, we may conclude that $\lambda^{\prime} \cdot x=0$ so that $\lambda^{\prime} \cdot \widehat{x} \geqslant \lambda^{\prime} \cdot z^{\prime}$ for all $z^{\prime} \in \mathbb{R}^{N}$ with $\bar{g}\left(z^{\prime}\right)=0$. Thus, $\lambda^{\prime}$ is proportional to $\nabla \bar{g}(z)$.

Now, $\widehat{P}$ is p-smooth, then $\widetilde{P}=\widehat{P}$ satisfies the desired properties. Hence, we may assume that $\widehat{P}$ is not p-smooth. We now construct a p-smooth feasible set $\widetilde{P}$ with $\frac{1}{2} \widehat{P} \subseteq \widetilde{P} \subseteq \widehat{P}$. Note that $\frac{1}{2} \widehat{P}$ is a smooth convex feasible set that contains $y=0$ in its boundary. Moreover, $g^{\lambda^{y}, \frac{1}{2}} \widehat{P}(z)=\frac{\bar{g}(2 z)}{2}$ for all $z \in \mathbb{R}^{N}$. For $i \in N$, let $X^{i}=\left\{x \in X \mid \frac{\partial \bar{g}}{\partial z_{i}}(x) \leqslant \frac{1}{2}\right\}$. As $\widehat{P}$ is not p-smooth, by Remark 2.2 (2.7), a careful inspection of (6.8) shows that $X^{i} \neq \emptyset$ for all $i \in N$. Let $x, x^{\prime} \in X^{i}$ such that $\left\|x^{\prime}\right\| \geqslant\|x\|$. As $\widehat{g}$ is convex and $\bar{g}(z)=\widehat{g}(\|z\|)$ for all $z \in X, \bar{g}\left(x^{\prime}\right) / 2-\bar{g}\left(x^{\prime} / 2\right) \geqslant \bar{g}(x) / 2-\bar{g}(x / 2)$. As $X^{i}$ is closed, we may conclude that

$$
\alpha_{i}=\min _{x \in X^{i}} \frac{\bar{g}(x)}{2}-\bar{g}\left(\frac{x}{2}\right)
$$

exists. Moreover, by smoothness of $\widehat{P}, \alpha^{i}>0$. Finally, by (6.6) we may conclude that $\alpha_{i}=\alpha_{j}$ for all $i, j \in N$. Now we are ready to construct $\widetilde{P}$. Let $\varepsilon \leqslant \min \left\{\frac{\alpha_{i}}{|N|}, \frac{1}{2|N|}\right\}$ for all $i \in N$ and define

$$
Q=\left\{y \in \mathbb{R}^{N} \mid y^{+}(N) \leqslant \varepsilon \frac{y^{-}(N)}{1+y^{-}(N)}\right\},
$$

where $x^{+}$and $x^{-}$denote the positive and negative part of any $x \in \mathbb{R}^{N}$, i.e., $x^{+}, x^{-} \in \mathbb{R}_{+}^{N}$ such that $x=x^{+}-x^{-}$. Note that $Q$ is convex and feasible and that $Q \subseteq\left(-\mathbb{R}_{+}^{N}\right)+\left\{\varepsilon \chi^{N}\right\}$. Let $\widetilde{P}=\frac{1}{2} \widehat{P}+Q$. It is straightforward to verify that $\widetilde{P}$ is closed so that $\widetilde{P}$ is feasible. We conclude that $Q$ satisfies the assumptions in (2) of Remark 6.1. It suffices to show that $\widetilde{P} \subseteq \widehat{P}$. Let $z \in \partial \widetilde{P}$. Then there exist $x \in \partial\left(\frac{1}{2} \widehat{P}\right), y \in \partial Q$, and $\lambda \in \mathbb{R}_{+}^{N}$ with $\lambda(N)=1$ so that $z=x+y$ and $\frac{1}{2} \widehat{P} \subseteq\left\{x^{\prime} \in \mathbb{R}^{N} \mid x^{\prime} \cdot \lambda \leqslant x \cdot \lambda\right\}$ and $Q \subseteq\left\{y^{\prime} \in \mathbb{R}^{N} \mid y^{\prime} \cdot \lambda \leqslant y \cdot \lambda\right\}$. If $|N| \min _{i \in N} \lambda_{i}>\frac{1}{2}$, then

$$
\begin{aligned}
\lambda \cdot y & \leqslant \frac{-y^{-}(N)}{2|N|}+y^{+}(N) & \leqslant \frac{-y^{-}(N)}{2|N|}+\varepsilon \frac{y^{-}(N)}{1+y^{-}(N)} \\
& \leqslant y^{-}(N)\left(\frac{\varepsilon}{1+y^{-}(N)}-\frac{2}{2|N|}\right) & \leqslant y^{-}(N)\left(\varepsilon-\frac{1}{2|N|}\right) \leqslant 0=\lambda \cdot 0
\end{aligned}
$$

so that $y=0$ and, hence, $z \in \widehat{P}$. If $|N| \min _{i \in N} \lambda_{i} \leqslant \frac{1}{2}$, then, by construction, $x+\varepsilon \chi^{N} \in \widehat{P}$. As $y \leqslant \varepsilon \chi^{N}, x+y \in \widehat{P}$.

q.e.d. 
Corollary 6.3 Let $P \subseteq \mathbb{R}^{N}$ be a uniformly p-smooth feasible set and $y \in \partial P$. Then there exists a uniformly p-smooth feasible set $\widetilde{P} \subseteq \mathbb{R}^{N}$ that satisfies (6.1) through (6.3).

Proof: By Lemma 2.1 there exists a p-smooth feasible set $\widetilde{P}$ with the desired properties.

We shall now modify $\widetilde{P}$ as follows: For $\mu>0$ let

$$
X_{\mu}=\left\{z \in \mathbb{R}^{N} \mid\left(\mu \chi^{N \backslash\{i\}}+(1-(|N|-1) \mu) \chi^{\{i\}}\right) \cdot z \leqslant 0 \forall i \in N\right\} .
$$

As $P$ is uniformly p-smooth, for any $\mu>0$ with $\mu<\min \left\{\lambda_{i}^{y} \mid i \in N\right\}$,

$$
P+X_{\mu} \subseteq P
$$

Define $\widehat{P}=\widetilde{P}+X_{\mu}$. As a sum of two convex sets, $\widehat{P}$ is convex. By (6.9) and (6.7), $\widehat{P} \subseteq P$, and $y \in \widehat{P}$, because $y \in \widetilde{P}$. As $X_{\mu}$ is a convex cone, $\{x\}+X_{\mu} \subseteq \widehat{P}$ for all $x \in \widetilde{P}$. As $X_{\mu} \supseteq-\mathbb{R}_{+}^{N}, \widehat{P}$ is comprehensive.

We now show that $\widehat{P}$ is closed. Let $\left(\widehat{x}^{t}+x^{t}\right)_{t \in \mathbb{N}}$ be a convergent $\mathbb{R}^{N}$-sequence such that $\widetilde{x}^{t} \in \widetilde{P}$ and $x^{t} \in X_{\mu}$ for all $t \in \mathbb{N}$. It remains to prove that $z=\lim _{t \rightarrow \infty} \widehat{x}^{t}+x^{t} \in \widehat{P}$. If $z^{\prime} \in \mathbb{R}^{N}$ such that $\widehat{x}^{t}+x^{t} \geqslant z^{\prime}$ for all $t \in \mathbb{N}$, then $\widehat{x}^{t} \in\left\{z^{\prime}\right\}-X_{\mu}$ forall $t \in \mathbb{N}$. As $\mu<\lambda_{i}^{y}$ for all $i \in N$, $\left\{x \in \mathbb{R}^{N} \mid \lambda^{y} \cdot x \leqslant \lambda^{y} \cdot y\right\} \cap\left(\left\{z^{\prime}\right\}-X_{\mu}\right)$ is compact. As $\widetilde{P} \subseteq\left\{x \in \mathbb{R}^{N} \mid \lambda^{y} \cdot x \leqslant \lambda^{y} \cdot y\right\}$, $\widetilde{P} \cap\left(\left\{z^{\prime}\right\}-X_{\mu}\right)$ is also compact. Hence, $\left(\widehat{x}^{t}\right)_{t \in \mathbb{N}}$ has a convergent subsequence, let us say, is convergent itself. We conclude that $\left(x^{t}\right)_{t \in \mathbb{N}}$ is also convergent. As $\widetilde{P}$ and $X_{\mu}$ are closed, $\lim _{t \rightarrow \infty} \widehat{x}^{t} \in \widetilde{P}$ and $\lim _{t \rightarrow \infty} x^{t} \in X_{\mu}$ so that $z=\lim _{t \rightarrow \infty} \widehat{x}^{t}+\lim _{t \rightarrow \infty} x^{t} \in \widehat{P}$. Hence $\widehat{P}$ is feasible and Remark 6.1 finishes the proof.

q.e.d

\section{References}

Aumann, R. J. (1985): "An axiomatization of the non-transferability utility value", Econometrica, 53, $599-612$.

Conley, J. P., And S. Wilkie (1996): "An extension of the Nash bargaining solution to nonconvex problems", Games and Economic Behavior, 13, 26 - 38.

Herrero, M. J. (1989): "The Nash program: non-convex bargaining problems", Journal of Economic Theory, pp. $266-277$.

Mariotti, M. (1998): "Extending Nash's axioms to nonconvex problems", Games and Economic Behavior, 22, $377-383$.

Mariotti, M., And A. Villar (2005): "The Nash rationing problem", International Journal of Game Theory, 33, 367 - 377.

Maschler, M., and G. Owen (1989): "The consistent Shapley value for hyperplane games", International Journal of Game Theory, 18, 389 - 407. 
(1992): "The consistent Shapley value for games without side payments", in Rational Interaction: Essays in Honor of John Harsanyi, ed. by R. Selten, pp. 5 - 12, New York. Springer-Verlag.

NAsh, J. F. (1950): "The bargaining problem", Econometrica, 18, 155 - 162.

Peleg, B., P. Sudhölter, and J. M. Zarzuelo (2012): "On the impact of independence of irrelevant alternatives: the case of two-person NTU games", SERIEs, 3, $143-156$.

Peters, H., and D. Vermeulen (2010): "WPO, COV and IIA bargaining solutions for nonconvex bargaining problems", International Journal of Game Theory, online first, 34 pp.

Rubinstein, A., And L. Zhou (1999): "Choice problems with a 'reference' point", Mathematical Social Sciences, 37, 205 - 209.

Shapley, L. S. (1969): "Utility comparisons and the theory of games", in La Decision, aggregation et dynamique des ordres de preference, pp. 251 - 263, Paris. Edition du Centre National de le Recherche Scientifique.

Zhou, L. (1996): "The Nash bargaining theory with non-convex problems", Econometrica, 65, $681-685$. 